\title{
First Run II results from ALICE
}

\author{
Alberica Toia ${ }^{1,2, a}$ \\ on behalf of the ALICE Collaboration \\ ${ }^{1}$ Goethe University of Frankfurt \\ ${ }^{2} \mathrm{GSI}$
}

\begin{abstract}
The ALICE Collaboration is collecting data with both Minimum Bias and Muon triggers with pp collisions at $\sqrt{s}=13 \mathrm{TeV}$ in the ongoing LHC Run II. An excellent performance of tracking and PID in the central barrel and in the muon spectrometer has been obtained. First results on the charged-particle pseudorapidity density and on identified particle transverse momentum spectra at $\sqrt{s}=13 \mathrm{TeV}$ is presented.
\end{abstract}

\section{Introduction}

After a two-year long shutdown, the Large Hadron Collider (LHC) at CERN restarted its physics programme in June 2015 with proton-proton collisions at $\sqrt{s}=13 \mathrm{TeV}$, the highest centre-of-mass energy reached so far in laboratory.

The charged particle multiplicity density is a measure of the initial energy density and provides information on the dynamics of the soft particle production and its relation to the initial collision geometry. Particle production at collider energies originates from the interplay of perturbative (hard) and non-perturbative (soft) QCD processes. Soft scattering processes and parton hadronisation dominate the bulk of particle production at low transverse momenta and can only be modelled phenomenologically. Hence, these measurements provide constraints for a better tuning of models and event generators for hadron-collider and cosmic-ray physics. In addition, they are valuable reference data to study nuclear effects in nucleus-nucleus and proton-nucleus collisions, in particular allowing one to measure the nuclear modification factors $R_{\mathrm{AA}}$ and $R_{\mathrm{pA}}$, respectively, of the transverse-momentum spectra.

To investigate particle production mechanisms, ALICE has extensively studied the first moment, $\left\langle p_{\mathrm{T}}\right\rangle$, of the charged-particle transverse momentum spectrum and its correlation with the chargedparticle multiplicity $N_{\text {ch }}$. This has been studied by many experiments at hadron colliders covering collision energies from $\sqrt{s}=31 \mathrm{GeV}$ up to $7 \mathrm{TeV}$. All experiments observed an increase of $\left\langle p_{\mathrm{T}}\right\rangle$ with $N_{\text {ch }}$ in pp collisions, which could be reproduced in PYTHIA only including color reconnections in the hadronization and which was recognized as a hint of coherence in a high multiplicity environment [1].

\section{ALICE in Run II}

The ALICE apparatus and its performance in Run I are presented in detail elsewhere [2]. Here we only briefly mention the upgrades set up for Run II. More details are presented in [3]. During the

\footnotetext{
a e-mail: alberica.toia@ cern.ch
} 
shut-down the construction and installation of the TRD was completed, so that it now has full central barrel acceptance. The full TRD coverage provides an improved momentum resolution for tracking and a uniform electron and hadron identification.

The detectors located outside TOF used by two electromagnetic calorimeters with thicknesses of 1 nuclear interaction length, the high-resolution lead-tungsten PHOS and the large-acceptance leadscintillator EMCal, have been upgraded. The EMCal was extended with the installation of eight (six full-size and two one-third-size) super modules, forming the so-called dijet calorimeter (DCal) backto-back to the existing EMcal. The PHOS trigger electronics was modified so that the PHOS and Dcal essentially act as a single calorimeter, able to measure both single shower, as well as jet triggers.

A new subsystem, the ALICE diffractive (AD) detector, has been installed during LS1 to enhance the capabilities of the experiment to tag diffractive events. The AD detector consists of two double layers of scintillation counters, sensitive to charged particles with $p_{\mathrm{T}}$ down to $20 \mathrm{MeV} / \mathrm{c}$, placed far away from the interaction region on both sides, one in the ALICE cavern (at $\mathrm{z}=17.0 \mathrm{~m}$ ) and one in the LHC tunnel (at $\mathrm{z}=-19.5 \mathrm{~m}$ ), with a pseudorapidity coverage of $4.9<\eta<6.3$ and $-7.0<\eta<-4.8$, respectively.

The TPC has a $90 \mathrm{~m}^{3}$ drift volume and is divided into two parts by the central cathode, which is kept at $-100 \mathrm{kV}$. The end plates are equipped with multiwire proportional chambers (MWPC). The gas mixture of the TPC was changed from $\mathrm{Ne}-\mathrm{CO}_{2}$ (90:10) to $\mathrm{Ar}-\mathrm{CO}_{2}$ (88:12) for Run II to ensure a more stable response for the high particle flux generated during $\mathrm{p}-\mathrm{Pb}$ and $\mathrm{Pb}-\mathrm{Pb}$ running without significant degradation of momentum resolution at the low $p_{\mathrm{T}}$.

\section{Data Analysis}

We present the pseudorapidity $(\eta)$ and transverse-momentum $\left(p_{\mathrm{T}}\right)$ distributions of primary charged particles measured in proton-proton collisions at the centre-of-mass energy $\sqrt{s}=13 \mathrm{TeV}$ with the ALICE detector at the LHC [4]. Primary particles are defined as prompt particles produced in the collisions, including all decay products, with the exception of those from weak decays of strange particles. The pseudorapidity distribution is measured at central rapidity in $|\eta|<1.8$. The measurements reported here have been obtained for inelastic events (INEL) and events having at least one charged particle in the pseudorapidity interval $|\eta|<1$ (INEL $>0$ ). The transverse-momentum distribution of charged particles is measured in the range $0.15<p_{\mathrm{T}}<20 \mathrm{GeV} / \mathrm{c}$ and $|\eta|<0.8$ for INEL $>0$ events. The correlation between transverse momentum and particle multiplicity is also investigated by studying the evolution of the spectra with event multiplicity. The data have been compared to calculations from models commonly used at the LHC.

The data were collected after the startup of LHC Run II in June 2015, with beam conditions leading to a luminosity of about $5 \times 10^{27} \mathrm{~cm}^{-2} \mathrm{~s}^{-1}$, which corresponds to a rate of about $350 \mathrm{~Hz}$ for inelastic proton-proton collisions, and a small pile-up probability $\left(10^{-3}\right)$. The data were collected using a minimum-bias trigger requiring a hit in either the $\mathrm{V} 0$ scintillators or in the $\mathrm{AD}$ arrays. After removing background events with an offline selection based on the timing information from the $\mathrm{V} 0$ and the $\mathrm{AD}$ detectors, and the correlation between the number of clusters of pixel hits and the number of tracklets the remaining contamination is estimated with control triggers taken for various combinations of beam and empty buckets, to be negligible. The final minimum-bias sample used for the data analysis consist of about 1.5 million, which are further required to have a valid reconstructed vertex within $|z|<10$ $\mathrm{cm}$, to ensure uniform acceptance.

For the corrections we use Monte Carlo events from the PYTHIA 6 (Perugia-2011) event generator with particle transport performed via a GEANT3 simulation of the ALICE detector. 


\subsection{Pseudorapidity Distribution}

The measurement of the charged-particle pseudorapidity distribution is performed using short track segments, termed "tracklets". Tracklet candidates are formed using the position of the primary vertex and a pair of hits, one in each SPD layer. The primary vertex position is extracted by correlating hits in the two SPD layers. This technique effectively allows to reconstruct charged particles with $p_{\mathrm{T}}$ above the $50 \mathrm{MeV} / \mathrm{c}$ cut-off determined by particle absorption in the material. The vertex position requirement results in an effective $|\eta|<1.8$ coverage. The charged particle pseudorapidity density is obtained from the measured distribution of tracklets, corrected for the acceptance and efficiency for a primary particle to produce a tracklet, and the contamination of reconstructed tracklets from combinations of hits not produced by the same primary particle. Both correction factors are determined as a function of the $z$ position of the primary vertex and the pseudorapidity of the tracklet from detector simulations. Differences in strange-particle content between data and simulations, observed at lower beam energies, are taken into account by scaling the strangeness production in the Monte Carlo event sample.

\subsection{Momentum Distribution}

The transverse-momentum distribution is measured from tracks reconstructed using the information from the Inner Tracking System (ITS) and the Time Projection Chamber (TPC) detectors. Candidate tracks are selected with cuts on the number of space points used for tracking and on the quality of the track fit, as well as on the distance of closest approach to the reconstructed vertex, which result in an effective aceptance of $|\eta|<0.8$. The efficiency for track reconstruction is calculated with PYTHIA 6, and, since it depends on the particle type, a correction is applied to reproduce the spectra of strange particles measured by ALICE in minimum-bias pp collisions at $\sqrt{s}=7 \mathrm{TeV}$. Finally, the residual contamination from secondary particles, estimated from Monte Carlo simulations, is subtracted from the spectrum.

Various are the contributions to the systematic uncertainties of these analyses. One of the main contributions to the normalisation of the results comes from the limited knowledge of cross-sections and kinematics of diffractive processes, not yet measured at $\sqrt{s}=13 \mathrm{TeV}$, and therefore solely based on previous experimental data at lower collision energies and simulations with Monte Carlo event generators. For the transverse-momentum distribution the dominant sources of uncertainty are the track selections, the efficiency corrections and, for low $p_{\mathrm{T}}$, the contamination from weak decays of strange hadrons. For the pseudorapidity distribution the dominant sources of uncertainty are the detector acceptance and efficiency, the correction due to $p_{\mathrm{T}}$ extrapolation down to zero, and also the contamination from weak decays of strange hadrons. Other minor systematic uncertainties also arise from the knowledge of the material budget, and of the particle composition.

\section{Results}

Figure 1, left panel, shows the average charged-particle density distribution $\mathrm{d} N_{\mathrm{ch}} / \mathrm{d} \eta$ measured in INEL and INEL $>0$ events in the pseudorapidity range $|\eta|<1.8$. Also shown in the Figure are the results recently published by the CMS Collaboration for inelastic collisions, which agree, within the uncertainties, with the ALICE measurement. We compared our measurement to Monte Carlo calculations performed with PYTHIA 6 (Perugia-2011), PYTHIA 8 (Monash-2013) and EPOS LHC in both the INEL and INEL $>0$ event classes. PYTHIA 6 calculations are in better agreement with the data than PYTHIA 8 in both classes. EPOS LHC calculations are sligthly higher than the data both in INEL and INEL $>0$ events. 

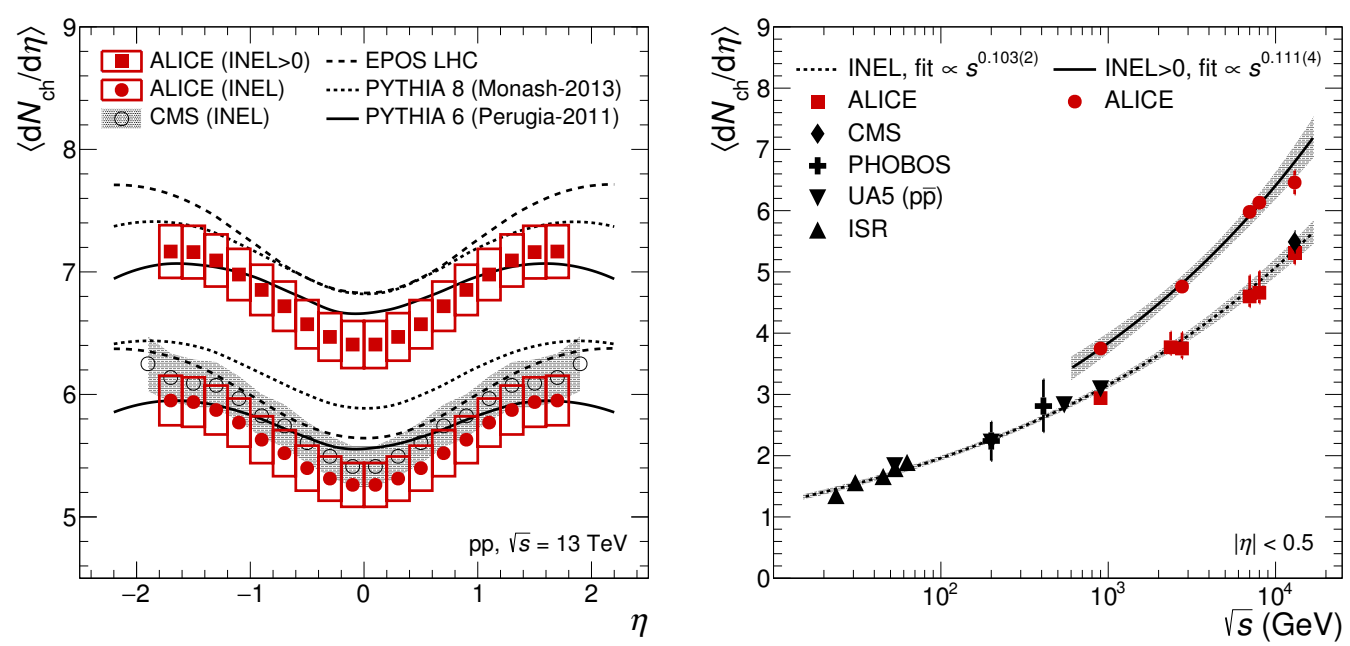

Figure 1. left: Average pseudorapidity density of charged particles as a function of eta produced in pp collisions at $\sqrt{s}=13 \mathrm{TeV}$. The ALICE results are shown in the normalisation classes INEL and INEL $>0$ and compared to Monte Carlo calculations and to the results from the CMS Collaboration. The uncertainties are the quadratic sum of statistical and systematic contributions. Right: Charged-particle pseudorapidity density measured in the central pseudorapidity region $|\eta|<0.5$ for INEL and INEL $>0$ events. The uncertainties are the quadratic sum of statistical and systematic contributions. The lines are power-law fits of the energy dependence of the data and the grey bands represent the standard deviation of the fits.

In the right panel of the Figure we show a compilation of results on pseudorapidity density of charged particles measured at central rapidity $(|\eta|<0.5)$ at different proton-proton collider energies. The energy dependence of $\mathrm{d} N_{\mathrm{ch}} / \mathrm{d} \eta$ is parametrised by the power law function fitted to data. Although the fit results assume that uncertainties at different centre-of-mass energies are independent, which is not strictly the case, the new measuremnt of Run II seems to be in line with the expectations from the data measured at lower energy.

Figure 2 presents the measured $p_{\mathrm{T}}$ spectrum and its comparison with calculations with PYTHIA 6 (Perugia-2011), PYTHIA 8 (Monash-2013) and EPOS LHC. For bulk particle production, the mechanism of colour reconnection is an important one in the PYTHIA models. EPOS is a model based on the Gribov-Regge theory at parton level. Collective (flow-like) effects are incorporated in the EPOS3 version and treated via parametrisations in the EPOS LHC version. These event generators, benefitting from the tuning performed on the LHC data in Run I, describe the $p_{\mathrm{T}}$ spectrum reasonably well, although not in detail. It is interesting to note that both PYTHIA 8 and EPOS LHC models show a similar pattern in the ratio to data with discrepancies up to $20 \%$ and that PYTHIA 6 overestimates particle production at high $p_{\mathrm{T}}$.

The right panel of the Figure shows the ratio of transverse-momentum spectra of charged particles at $\sqrt{s}=13 \mathrm{TeV}$ and $7 \mathrm{TeV}$ [5], properly re-normalized in the INEL $>0$ event class. As expected, the spectrum is significantly harder at $\sqrt{s}=13 \mathrm{TeV}$ than at $\sqrt{s}=7 \mathrm{TeV}$. PYTHIA 6, PYTHIA 8 and EPOS LHC reproduce the trend observed in the data, but exhibit a slightly more pronounced hardening with energy in the region of a few $\mathrm{GeV} / \mathrm{c}$. The effect appears to be more significant in PYTHIA 8 than in PYTHIA 6 and EPOS LHC. 

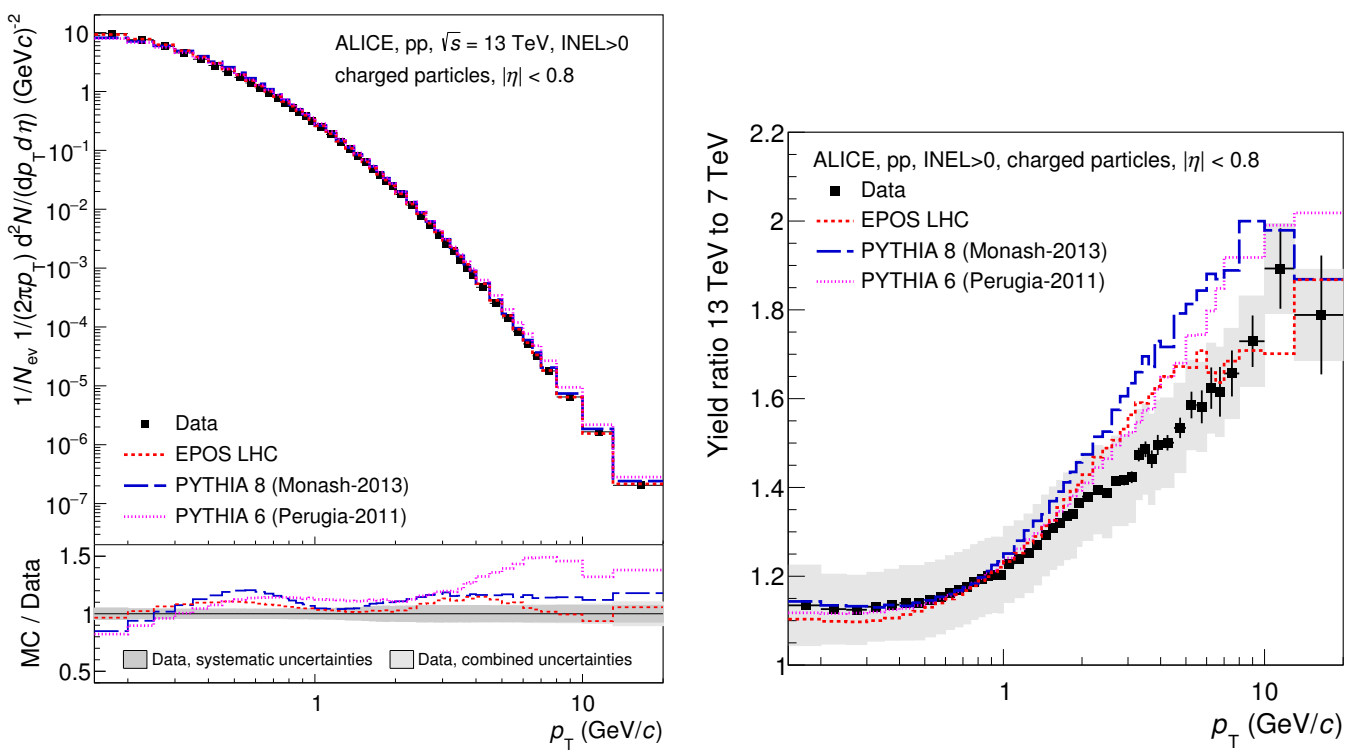

Figure 2. Left:Invariant charged-particle yield as a function of $p_{\mathrm{T}}$ normalised in the INEL $>0$ event class. The data are compared to Monte Carlo calculations. For the ratio of models (MC) and data (lower panel) the systematic and total uncertainties of the data are shown as grey bands. Right: Ratio of transverse-momentum spectra in INEL $>0$ events at $\sqrt{s}=13$ and $7 \mathrm{TeV}$. The boxes represent the systematic uncertainties, given by the quadratic sum of uncertainties at the two energies. The data are compared to Monte Carlo calculations.

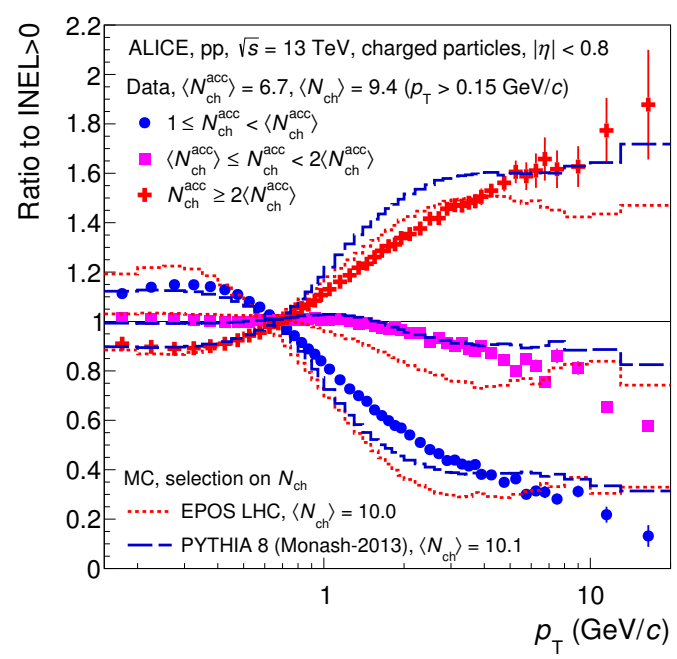

Figure 3. Ratios of transverse-momentum distributions of charged particles in three intervals of multiplicities to the respective one for inclusive (INEL $>0$ ) collisions. The spectra were normalized by the integral prior to division. The data are compared to Monte Carlo calculations. 
The correlation of the particle mean transverse momentum $\left(\left\langle p_{\mathrm{T}}\right\rangle\right)$ with the multiplicity of the event $\left(N_{\mathrm{ch}}\right)$ first observed at the SppS collider, has been studied by many experiments at hadron colliders in $\operatorname{pp}(\bar{p})$ covering collision energies from $\sqrt{s}=31 \mathrm{GeV}$ up to $7 \mathrm{TeV}$. The increase of $\left\langle p_{\mathrm{T}}\right\rangle$ with $N_{\text {ch }}$ in the central rapidity region observed in all experiments could be reproduced in the PYTHIA event generator only if a mechanism of hadronisation with colour reconnections (CR) is considered. Whether a connection exists between $\mathrm{CR}$ and flow in the sense of a genuine space-time evolution of the system is a subject of current investigation. A mechanism involving collective string hadronisation is also used in the EPOS model.

To investigate closely the changes of the spectral shape with multiplicity, we calculate ratio of spectra measured in three intervals of multiplicity to the inclusive (INEL $>0$ ) spectrum, shown in Figure 3. In this way, the systematic uncertainties of the measured spectra cancel out completely in the ratios. For this ratio, the spectra were normalised by the integral prior to dividing. The selection is performed on the multiplicity measured in the same kinematic region as the spectrum, $|\eta|<0.8$ and $0.15<p_{\mathrm{T}}<20 \mathrm{GeV} / \mathrm{c}$, using the uncorrected measured track multiplicity for data and the true value of $N_{\text {ch }}$ known in Monte Carlo events. The multiplicity cuts correspond to the average and twice the average value.

The data in the Figure show that the correlation of the spectrum with multiplicity is prominent for the whole $p_{\mathrm{T}}$ range and particularly stronger at high $p_{\mathrm{T}}$. This correlation is influenced by hard parton production, which, through fragmentation (leading to jets), contributes to charged-particle multiplicity and this contribution is increasing with the parton energy. The increase of $\left\langle p_{\mathrm{T}}\right\rangle$ with multiplicity, typically moderate, is most enhanced in the present analysis of spectra and multiplicity, measured in the same kinematic region, where jets presumably dominate the high-multiplicity domain.

The general features seen in the data, which are similar to those first seen $\sqrt{s}=0.9 \mathrm{TeV}$, are reproduced by PYTHIA 8 and EPOS LHC fairly well. Some discrepancies, of no more than 20\%, are noticeable in particular in the high $p_{\mathrm{T}}$ region. This is more prominent for EPOS LHC. It was shown earlier that both EPOS LHC and PYTHIA 8 reproduce well, although slightly overpredicting, the correlation of $\left\langle p_{\mathrm{T}}\right\rangle$ with $N_{\mathrm{ch}}$. The present data on spectral shape highlight some deficiencies in both models concerning the description of spectral shapes as a function of multiplicity.

\section{Conclusions}

In conclusions, measurement of the pseudorapidity and tranverse-momentum distribution of charged particles produced in proton-proton collisions at $\sqrt{s}=13 \mathrm{TeV}$ were presented. The $p_{\mathrm{T}}$ spectrum is significantly harder than at $\sqrt{s}=7 \mathrm{TeV}$ and its shape depends strongly on the multiplicity (in the same kinematic region). The correlation of the spectrum with multiplicity is prominent for all $p_{\mathrm{T}}$ and is observed to be stronger at high $p_{\mathrm{T}}$, influenced by hard parton production, which, through fragmentation (leading to jets), contributes to charged-particle multiplicity. All in all, there is a fair agreement of the data with expectations from lower energy extrapolations and with the Monte Carlo models used for comparison (PYTHIA6, PYTHIA8, EPOS-LHC).

\section{References}

[1] B. Abelev et al. (ALICE Collaboration), Phys. Lett. B 727 (2013) 371-380

[2] B. Abelev et al. (ALICE Collaboration), Int. J. Mod. Phys. A 29 (2014) 1430044

[3] J. Adam et al. (ALICE Collaboration), ALICE-PUBLIC-2015-004 (2015)

[4] J. Adam et al. (ALICE Collaboration), Phys. Lett. B 753 (2016) 319-329

[5] B. Abelev et al. (ALICE Collaboration), Eur. Phys. J. C73 no. 12 (2013) 